Supporting Information for the paper:

\title{
Metallacycloheptatrienes of Ir(III): Synthesis and Reactivity
}

\author{
Margarita Paneque, ${ }^{* \dagger}$ Cristina M. Posadas, ${ }^{\dagger}$ Manuel L. Poveda, ${ }^{, \dagger}$ Nuria Rendón, ${ }^{\dagger}$ \\ Laura. L. Santos, ${ }^{\dagger}$ Eleuterio Álvarez, ${ }^{\dagger}$ Verónica Salazar, ${ }^{\ddagger}$ Kurt Mereiter, ${ }^{\S}$ and Enrique \\ Oñate
}

Instituto de Investigaciones Químicas and Departamento de Química Inorgánica, Consejo Superior de Investigaciones Científicas (CSIC) and Universidad de Sevilla, Avenida Américo Vespucio 49, Isla de la Cartuja, 41092 Sevilla, Spain, Centro de Investigaciones Químicas, Universidad Autónoma del Estado de Hidalgo, Carretera Pachuca a Tulacingo, Pachuca, Hidalgo, México, Department of Chemistry, Vienna University of Technology, Getreidemarkt 9/164SC, A-1060 Vienna, Austria, and Instituto de Ciencia de Materiales de Aragón, CSIC and Universidad de Zaragoza, Plaza de San Francisco s/n, Zaragoza, Spain

Figure S1. ${ }^{1} \mathrm{H}$ NMR spectrum of compound $13\left(\mathrm{CDCl}_{3}\right)$.

Figure S2. ${ }^{13} \mathrm{C}\left\{{ }^{1} \mathrm{H}\right\}$ NMR spectrum of compound $\mathbf{1 3}\left(\mathrm{CDCl}_{3}\right)$.

Figure S3. ${ }^{1} \mathrm{H}$ NMR spectrum of compound $14\left(\mathrm{CD}_{3} \mathrm{OD}\right)$.

Figure S4. ${ }^{13} \mathrm{C}\left\{{ }^{1} \mathrm{H}\right\}$ NMR spectrum of compound $\mathbf{1 4}\left(\mathrm{CD}_{3} \mathrm{OD}\right)$.

Figure S5. ${ }^{1} \mathrm{H}$ NMR spectrum of compound $15\left(\mathrm{CDCl}_{3}\right)$.

Figure S6. ${ }^{13} \mathrm{C}\left\{{ }^{1} \mathrm{H}\right\}$ NMR spectrum of compound $\mathbf{1 5}\left(\mathrm{CDCl}_{3}\right)$.

Figure S7. ${ }^{1} \mathrm{H}$ NMR spectrum of compound $17\left(\mathrm{CD}_{2} \mathrm{Cl}_{2}\right)$.

Figure S8. ${ }^{13} \mathrm{C}\left\{{ }^{1} \mathrm{H}\right\}$ NMR spectrum of compound $17\left(\mathrm{CD}_{2} \mathrm{Cl}_{2}\right)$. 


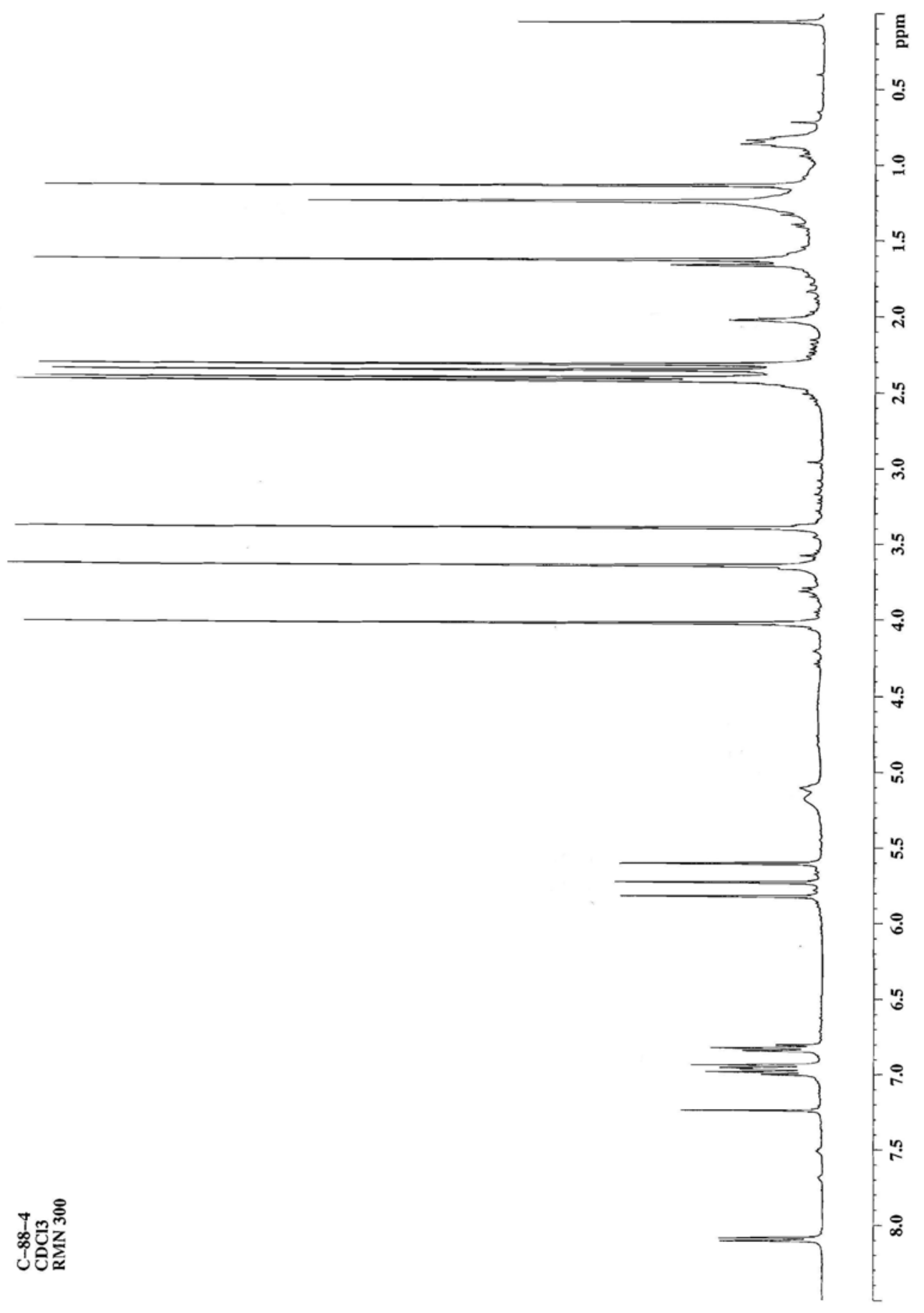

Figure S1. ${ }^{1} \mathrm{H}$ NMR spectrum of compound $\mathbf{1 3}\left(\mathrm{CDCl}_{3}\right)$. 


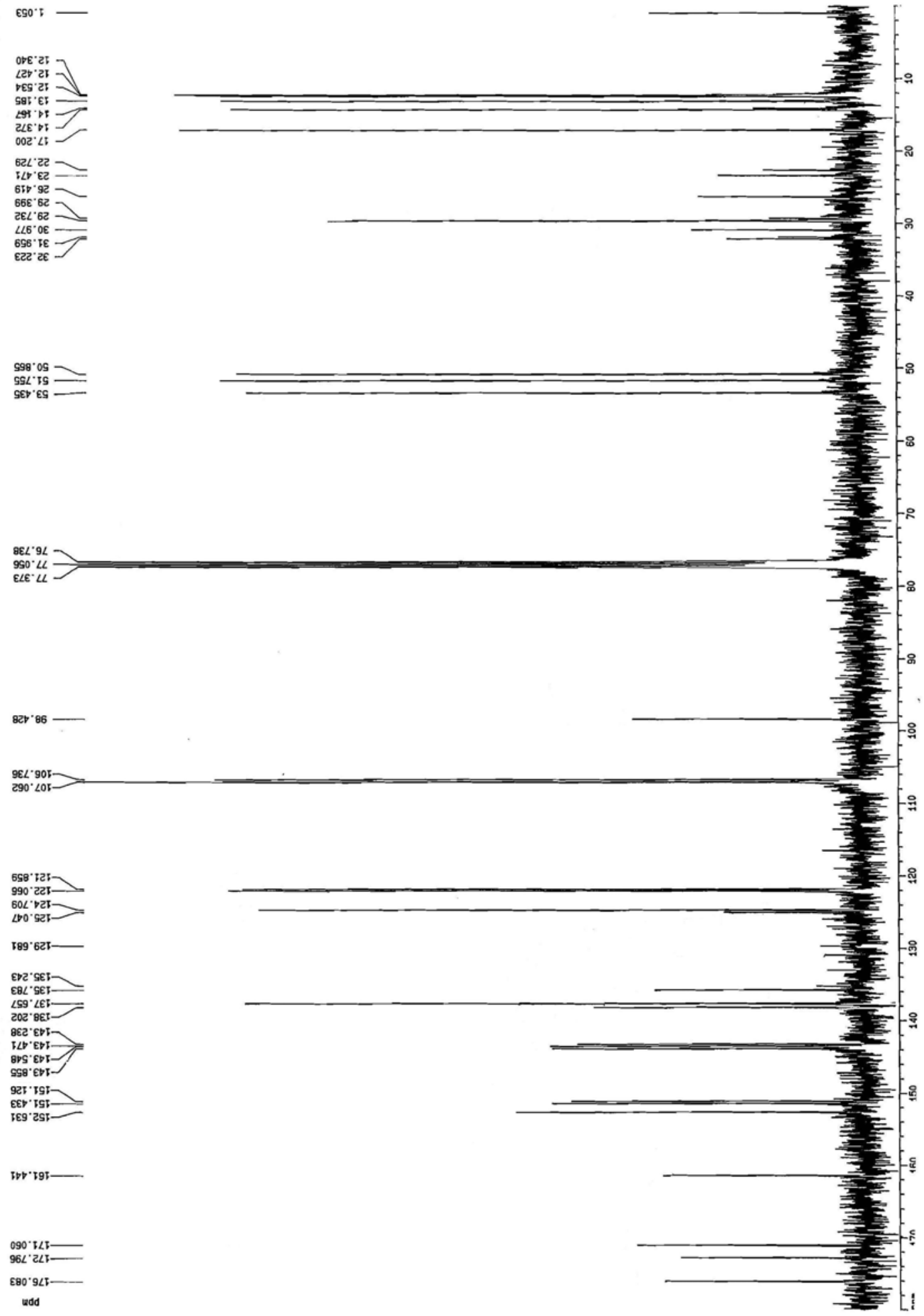

Figure S2. ${ }^{13} \mathrm{C}\left\{{ }^{1} \mathrm{H}\right\}$ NMR spectrum of compound $\mathbf{1 3}\left(\mathrm{CDCl}_{3}\right)$. 


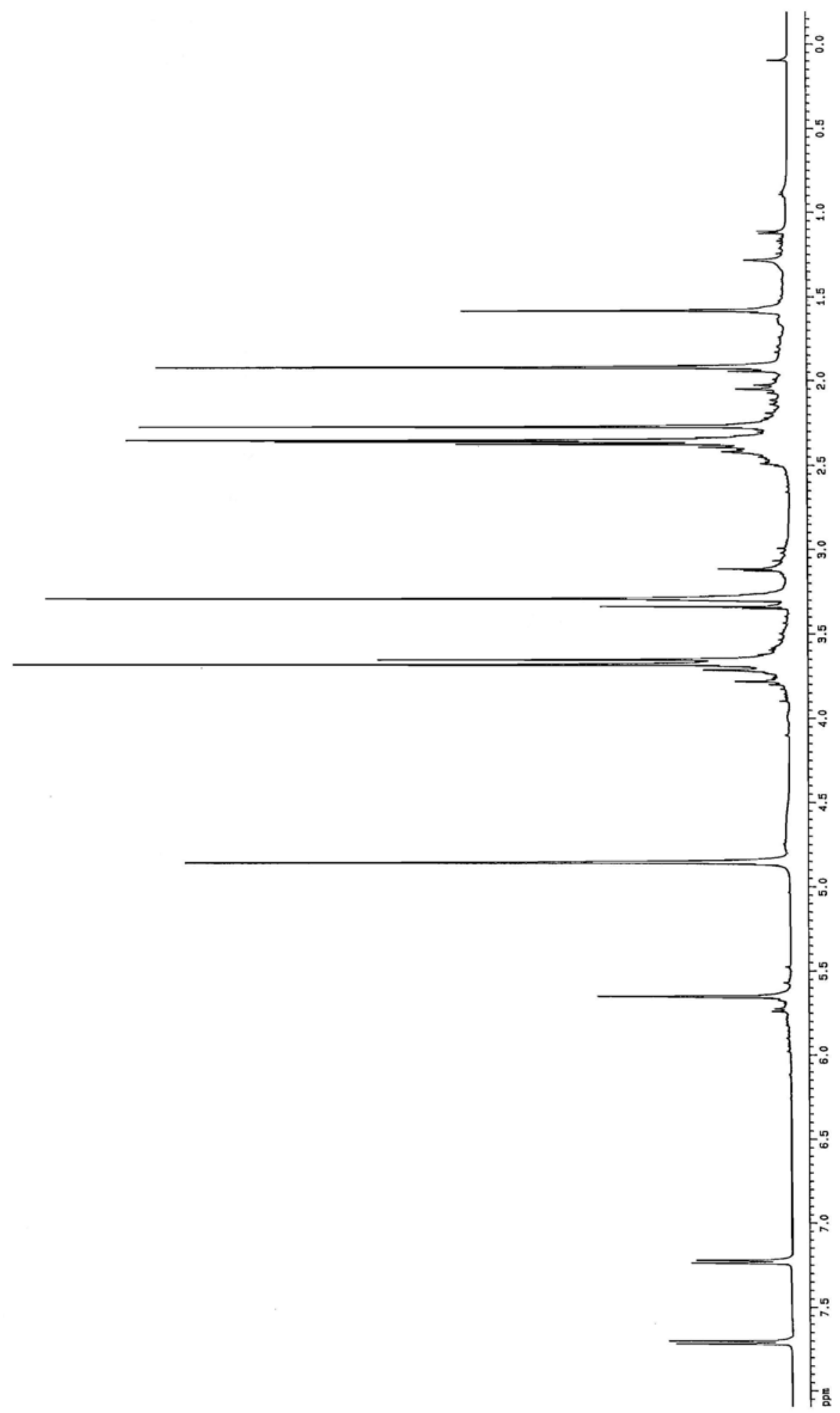

Figure S3. ${ }^{1} \mathrm{H}$ NMR spectrum of compound $14\left(\mathrm{CD}_{3} \mathrm{OD}\right)$. 
${ }_{400}^{\circ} \mathrm{CI}$

$58 C^{\circ} \circ 5$
$\angle B^{\circ} \rightarrow 5$

เ6อ' เา -

เธน'ยट -

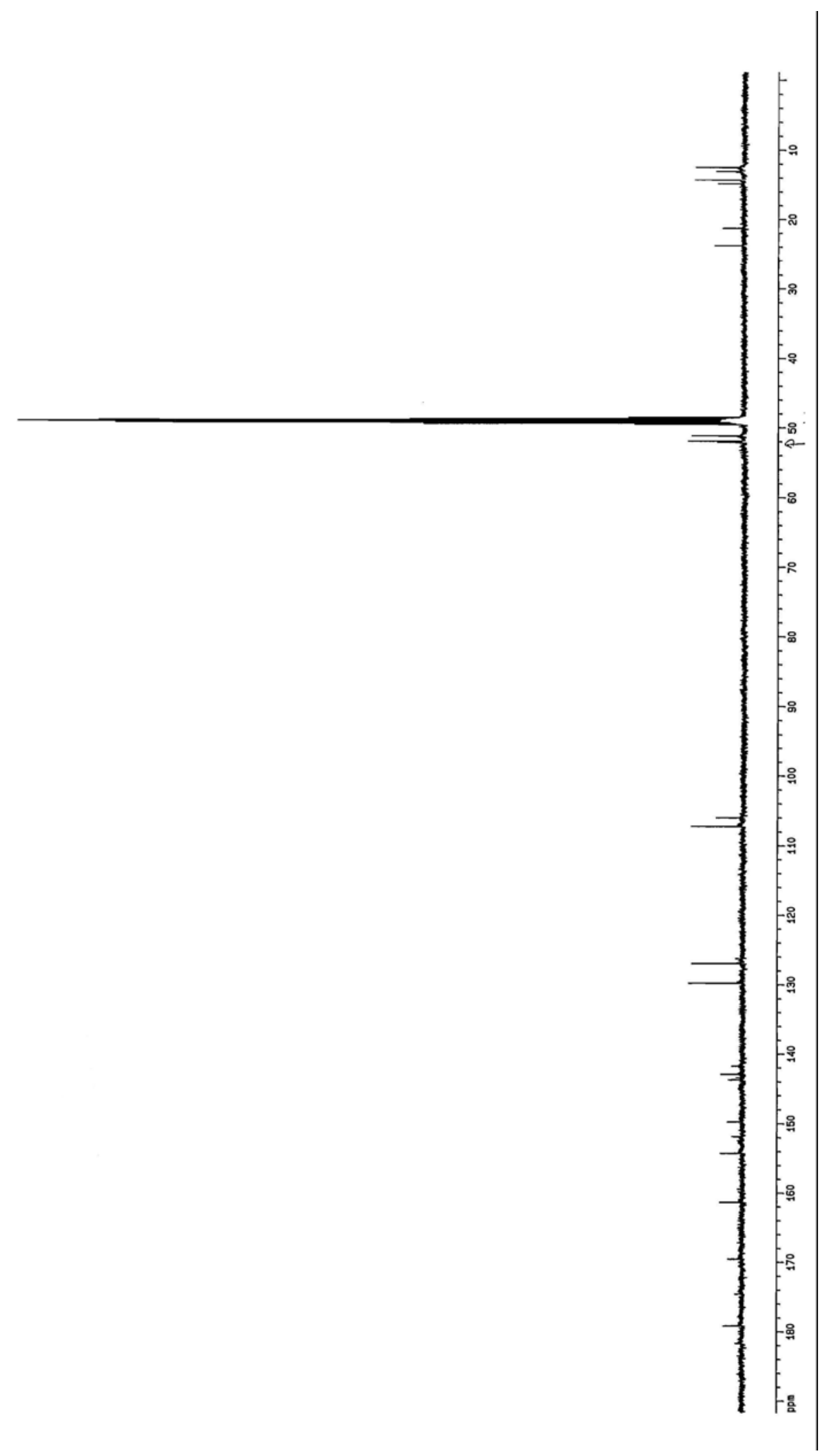

086 ' $005-$

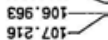

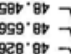

$\left.\angle E 6^{\circ} \theta b\right]$

$\angle 9 \Gamma^{\circ} 6 t>$

905' 6 ' $^{\circ}$ '

$\mathrm{sec}$ ' is

00\%'25]

092.925

${ }^{896} \cdot 925-$

$6 \angle 2.651-$

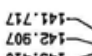

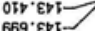

(1)

IS8 $\mathrm{tSt}-$

टहा"टS:-

จะย. เ $95-$

TWS' 695

E6S' $V$ LI-

$\angle E T \cdot 6 \angle L$

$099^{\circ} \mathrm{r} 8 \mathrm{i}-$

BLL. $\angle 85-$

wdd

Figure S4. ${ }^{13} \mathrm{C}\left\{{ }^{1} \mathrm{H}\right\}$ NMR spectrum of compound $\mathbf{1 4}\left(\mathrm{CD}_{3} \mathrm{OD}\right)$. 


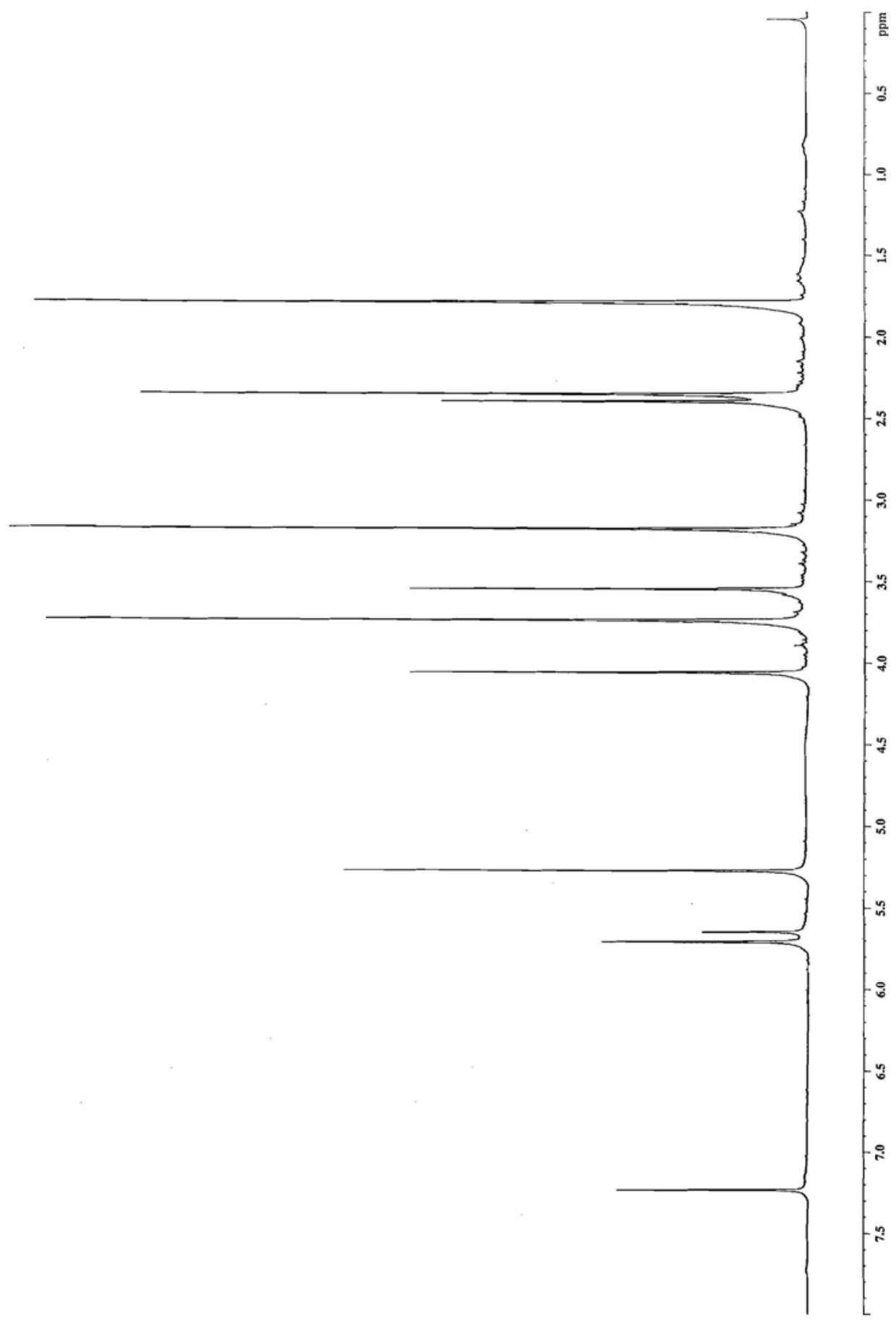

Figure S5. ${ }^{1} \mathrm{H}$ NMR spectrum of compound $\mathbf{1 5}\left(\mathrm{CDCl}_{3}\right)$. 

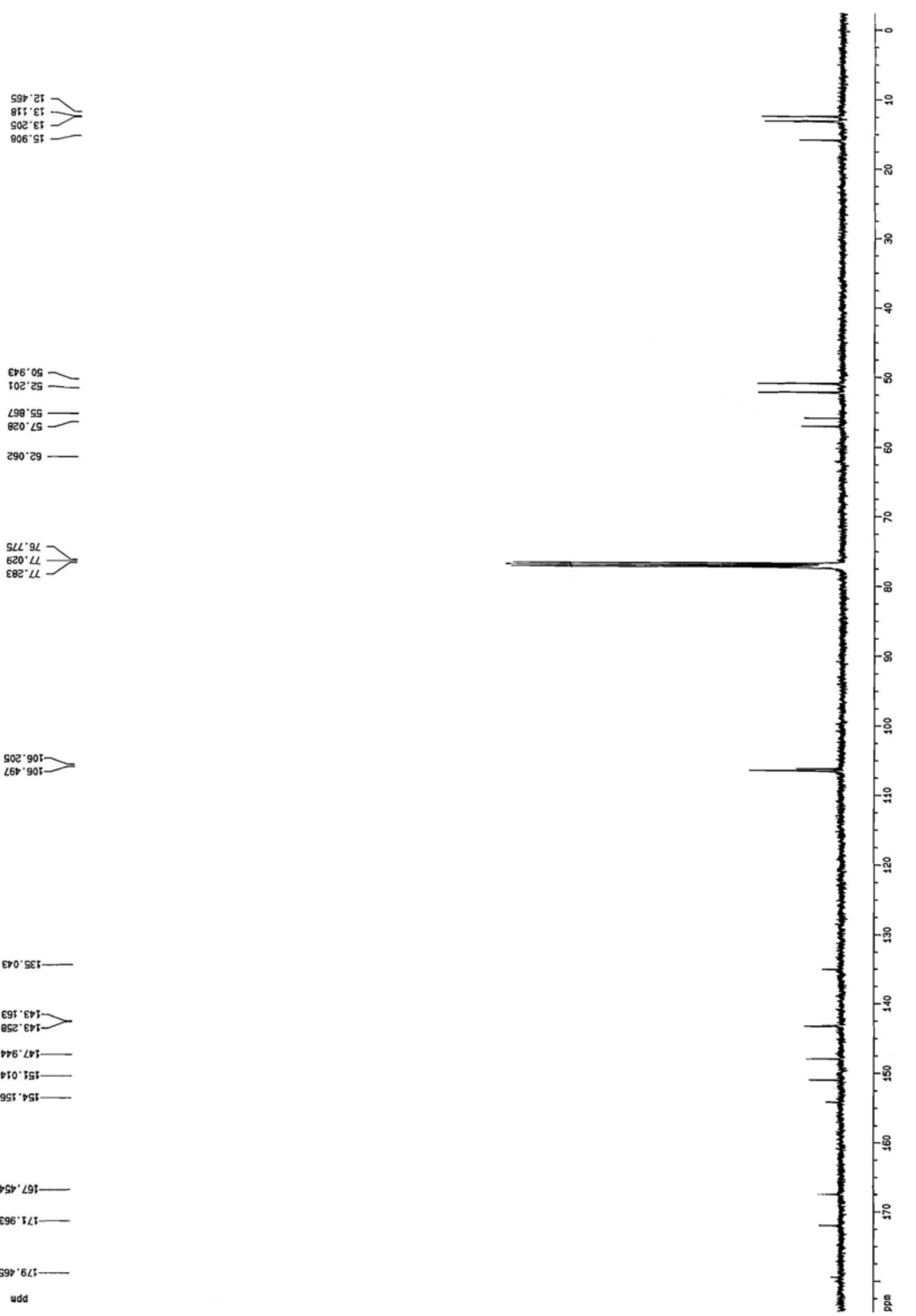

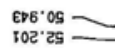

$\angle 90^{\circ} \mathrm{sS}=$

$290.29-$

$\frac{9 L \cdot 9 L}{620^{\circ}} \angle L>$

502 9005

egr' $E b 5$

$076 . \angle 05-$

9c5 '十95-

$\forall \$ D^{\circ} \angle 9 t-$

$696 \cdot 5 / 5-$

$598 * 6 \angle 5-$

udd

Figure S6. ${ }^{13} \mathrm{C}\left\{{ }^{1} \mathrm{H}\right\}$ NMR spectrum of $\mathbf{1 5}\left(\mathrm{CDCl}_{3}\right)$. 


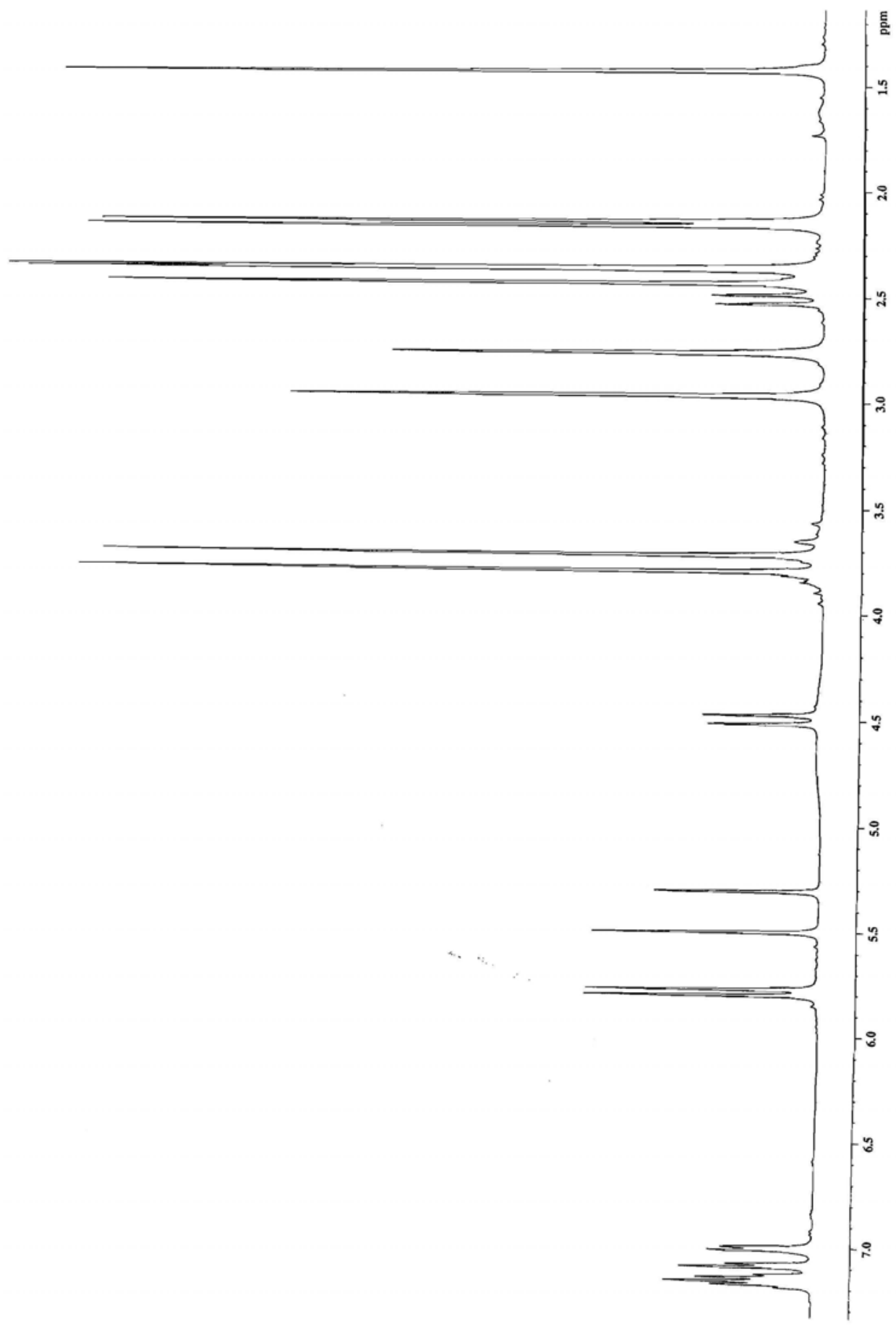

Figure S7. ${ }^{1} \mathrm{H}$ NMR spectrum of compound $17\left(\mathrm{CD}_{2} \mathrm{Cl}_{2}\right)$. 


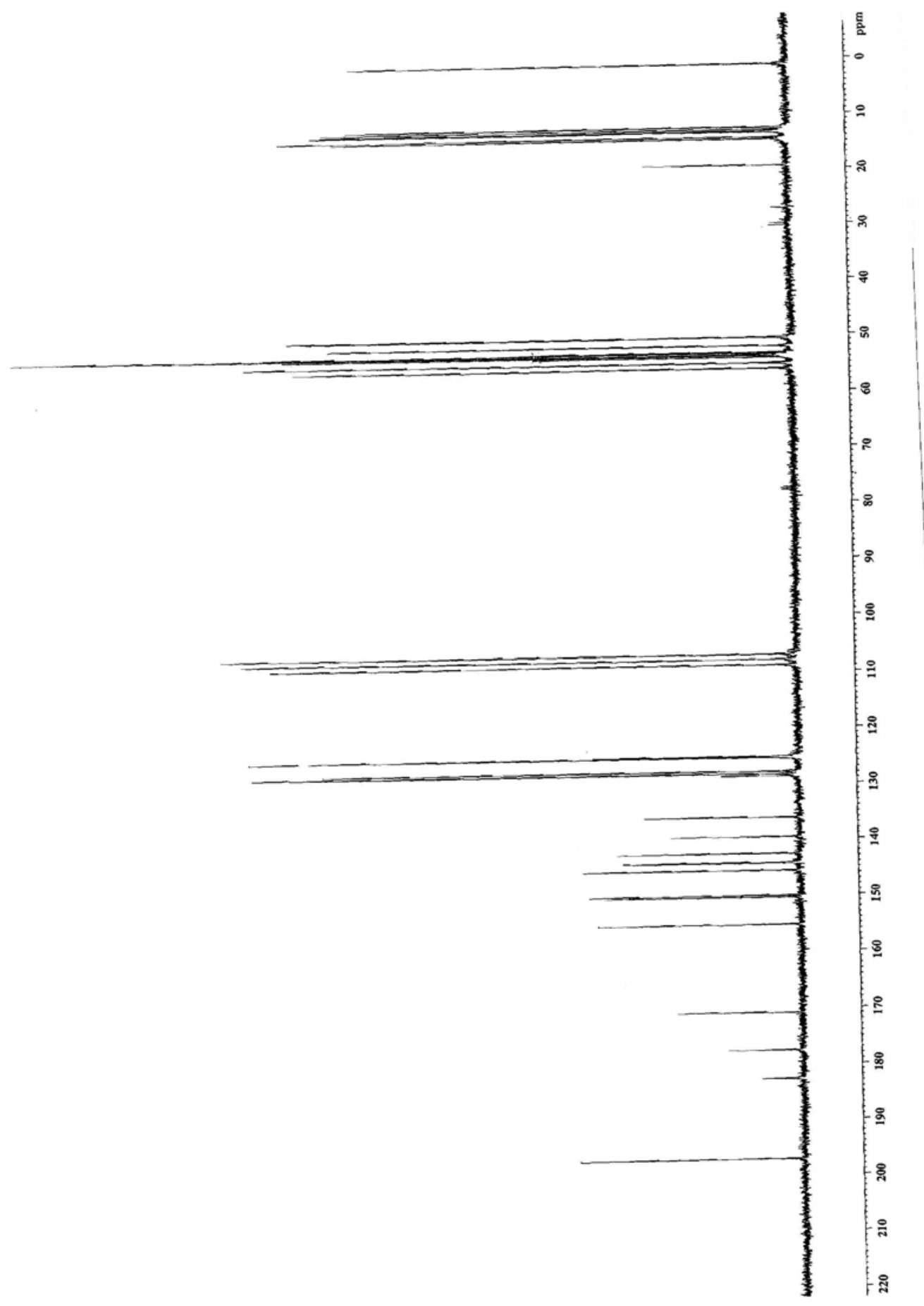

Figure 8. ${ }^{13} \mathrm{C}\left\{{ }^{1} \mathrm{H}\right\}$ NMR spectrum of compound $17\left(\mathrm{CD}_{2} \mathrm{Cl}_{2}\right)$. 